\title{
gifORSETI
}

\section{Establecimiento permanente: ¿es la sede de dirección un nuevo criterio de domicilio de las personas jurídicas?}

\author{
Fabio Taboada Pérez*
}

Resumen. - En el presente artículo, el autor reflexiona alrededor de incorporación del criterio de "sede de dirección efectiva" como un nuevo criterio para determinar el domicilio fiscal de las personas jurídicas. ¿Qué es una sede de dirección?, ¿es acaso que a través de la regulación de la figura de establecimiento permanente se ha creado un nuevo criterio para determinar el domicilio de las personas jurídicas de manera semejante al criterio sede de dirección efectiva?, son algunas de las preguntas a las que el autor dará respuesta, en el marco de la promulgación del Decreto Legislativo $\mathrm{N}^{\circ} 1424$.

\begin{abstract}
In this article, the author reflects on the incorporation of the criterion of "effective management headquarters" as a new criterion to determine the fiscal domicile of legal persons. What is an address? Is it possible that, through the regulation of the permanent establishment figure, a new criterion has been created to determine the domicile of legal entities in a manner similar to the effective management headquarters? This are some of the questions to which the author will respond, within the framework of the enactment of Legislative Decree $\mathrm{N}^{\mathrm{o}} 1424$.
\end{abstract}

* Abogado por la Pontifica Universidad Católica del Perú. Miembro del Colegio de Abogados de Lima. Asociado senior de PwC Perú. Miembro del Instituto Peruano de Derecho Tributario y de la Asociación Fiscal Internacional - Grupo Peruano. Miembro fundador del Grupo de Investigación en Derecho Internacional Tributario (GIDIT). 


\section{Introducción}

La Ley 30823, publicada el 19 de julio de 2018 en el Diario Oficial "El Peruano", delegó en el Poder Ejecutivo la facultad de legislar por el plazo de sesenta (60) días calendario en materia de gestión económica y competitividad, de integridad y lucha contra la corrupción, de prevención y protección de personas en situación de violencia y vulnerabilidad, y de modernización de la gestión del Estado. Dicha ley entró en vigencia el 20 de julio de 2018. El numeral 1(a) del artículo 2 de la Ley 30823 estableció que, en materia tributaria y financiera, se autorizaba a modificar la Ley del Impuesto a la Renta (LIR) respecto a los criterios de domicilio de las personas jurídicas.

Una vez publicada la Ley 30823, durante todo el plazo en que el Ejecutivo mantenía facultades legislativas, las cuales vencían el 17 de setiembre de 2018, se discutió en el ámbito académico acerca de la posible incorporación del criterio de "sede de dirección efectiva" como un nuevo criterio para determinar el domicilio fiscal de las personas jurídicas. La posibilidad de que se incorpore un criterio de sede de dirección efectiva no parecía alejada si tomamos en cuenta que es un criterio bastante desarrollado por la doctrina y que, además, es utilizado por algunos países de la región, como México y Colombia.

Sin embargo, para sorpresa de muchos, las facultades legislativas del Poder Ejecutivo vencieron el 17 de setiembre de 2018 y no se estableció ninguna modificatoria en cuanto a los criterios para determinar el domicilio de una persona jurídica (o al menos eso parecía).

Ahora bien, entre las normas emitidas por el Poder Ejecutivo, el Decreto Legislativo 1424, publicado el 13 de setiembre de 2018 y cuya entrada en vigencia será a partir del 1 de enero de 2019, incorporó el artículo 14-B a la LIR, en el cual se regula en toda su extensión la figura del establecimiento permanente.

Actualmente y hasta antes del 01 de enero de 2019, la expresión "establecimiento permanente" únicamente es mencionada en determinados artículos de la LIR, sobretodo en el artículo 7 (e), en el cual se indica que los establecimientos permanentes en el Perú de personas naturales o jurídicas no domiciliadas en el país se considerarán domiciliados en el país, en cuyo caso la condición de domiciliado alcanza al establecimiento permanente en cuanto a su renta de fuente peruana. Sin embargo, la regulación de la figura del establecimiento permanente como tal se encuentra contenida en el Reglamento de la LIR (RLIR).

Bajo dicho contexto, lo que hará el Decreto Legislativo 1424 a partir de su entrada en vigencia será establecer una nueva regulación acerca de la figura del establecimiento permanente y revestirla de jerarquía legal.

Ahora, un punto que inicialmente pasó de ser desapercibido es que el Decreto Legislativo 1424 en la definición general de un establecimiento permanente como lugar fijo de negocios, señaló una lista de qué lugares pueden llegar a constituir un 
establecimiento permanente bajo la definición general, entre las cuales se encuentra la "sede de dirección".

Nótese que la regulación de la figura de establecimiento permanente contenida en el RLIR, en cuanto a la definición general de qué es un establecimiento permanente, es idéntica a la que ha establecido el Decreto Legislativo 1424, con la única diferencia del listado de lugares, y en especial por el hecho de que el RLIR utiliza el término "centros administrativos", mientras que el Decreto Legislativo 1424 cambiará ese término por el de "sede de dirección".

Las preguntas caen de maduras: ¿qué motivó al Poder Ejecutivo a cambiar la expresión "centros administrativos" por "sede de dirección"? ¿Qué es una sede de dirección? Y, la pregunta más importante: ¿es acaso que a través de la regulación de la figura de establecimiento permanente se ha creado un nuevo criterio para determinar el domicilio de las personas jurídicas de manera semejante al criterio sede de dirección efectiva?

En efecto, se plantea la duda de si se habría agregado una regla complementaria a la del lugar de constitución, según la cual se consideraría domiciliada en el país a aquellas personas jurídicas constituidas en el exterior que tengan su sede de dirección en el país. Si fuera ello así, dicha persona jurídica generaría un establecimiento permanente en el país y se encontraría gravada por sus rentas de fuente peruana.

El presente trabajo aborda la problemática expuesta mediante un análisis de qué debe entenderse por "sede de dirección" y su distinción (si es que la hay) de otros criterios como el de "centros administrativos" o "sede de dirección efectiva"; y, si es que esta nueva regulación estaría en el fondo incorporando en el Perú un nuevo criterio para determinar el domicilio de las personas jurídicas.

\section{Centros administrativos vs sede de dirección}

El punto de partida para comprender una institución jurídica es realizar una interpretación literal de la norma. Si bien el intérprete puede utilizar otros métodos de interpretación y a veces es deseable que lo haga, no puede negarse que el intérprete empieza a interpretar una norma en base a la lectura de su propio texto y el significado que fluye de él.

En ese sentido, tratándose de la definición general de establecimiento permanente como lugar fijo de negocios, si la regulación contenida en el RLIR es idéntica a la regulación incorporada en la LIR, con la única excepción del listado de lugares, como es el caso de que la expresión "centros administrativos" se cambie por "sede de dirección", corresponde -como resulta lógico- desentrañar el significado de ambas expresiones.

La normativa del Impuesto a la Renta no contiene una definición acerca de qué debe entenderse por "centros administrativos" o "sede de dirección". Asimismo, 
de forma sorprendente, la Exposición de Motivos del Decreto Legislativo 1224 no señala a qué se debe el cambio de expresión. No obstante, resulta interesante destacar que, a lo largo de la Exposición de Motivos, se indica que las modificaciones tienen entre uno de sus objetivos el regular la figura del establecimiento permanente de acuerdo a los estándares internacionales (recomendaciones formuladas por la $\mathrm{OCDE}^{1}$ ) y la posición adoptada por el Estado peruano en los CDI suscritos.

Sobre este punto, nótese que el Modelo de CDI de la OCDE (MOCDE), así como los CDI suscritos por el Perú, en la cláusula de establecimiento permanente no utilizan la expresión "centros administrativos", sino que utilizan la expresión "sede de dirección", por lo que el hecho de que el Decreto Legislativo 1424 haya preferido utilizar la expresión "sede de dirección" obedecería al ánimo de uniformizar el lenguaje utilizado en la normativa del Impuesto a la Renta con el lenguaje utilizado por la OCDE (y que es utilizado por el Perú en sus respectivos CDI).

Ahora, ¿por qué el RLIR utiliza entonces la expresión "centros administrativos"? ¿Tiene un concepto distinto al de "sede de dirección" o son conceptos similares? Originalmente, al regularse la figura del establecimiento permanente en el RLIR, el legislador se inspiró en el Modelo de CDI de la Organización de las Naciones Unidas (MONU), el cual utilizaba la expresión "centros administrativos" en vez de la de "sede de dirección".

$\mathrm{Al}$ respecto, de los Comentarios al MONU (CMONU) se aprecia que la expresión "centros administrativos" no sería diferente de la expresión "sede de dirección" que utiliza el MOCDE, sino que son expresiones cuyo significado resultaría idéntico, tan así que para la ONU los comentarios que hace la OCDE sobre la "sede de dirección" son perfectamente aplicables para los "centros administrativos", siendo, por tanto, expresiones plenamente intercambiables.

En efecto, la identidad entre "centros administrativos" y "sede de dirección" había sido advertida tempranamente por el maestro Alfredo Gildemeister, al sostener lo siguiente:

“(..) podemos observar en primer lugar, que se menciona a los "centros administrativos". Dicho término, tomado del artículo 5.2.a de la Convención Modelo de las Naciones Unidas, reemplaza el de "sedes de dirección" empleado por el Modelo. En cambio, los Convenios del Pacto Andino se refieren a "una oficina o lugar de administración o dirección de negocios" en el inciso a) del artículo 7. Asimismo, la Ley peruana distingue entre centros administrativos y oficina. Se ha seguido pues el criterio utilizado tanto por el Modelo como por el de la ONU, eso es, entender que todo centro

1 Organización para la Cooperación y el Desarrollo Económicos. 
administrativo es una sede de dirección, distinto al concepto de oficina" 2 . (Énfasis agregado)

Teniendo en cuenta lo expuesto, podemos afirmar que el reemplazo de la expresión "centro administrativo" por el de "sede de dirección" responde a una adecuación de la normativa del Impuesto a la Renta al lenguaje utilizado y promovido por la OCDE, y que el Perú también adopta en los CDI suscritos. Si bien la legislación peruana había utilizado inicialmente la expresión "centro administrativo" en base al MONU, se entendía que el significado de dicha expresión era idéntico al de "sede de dirección", por lo que su reemplazo no acarrea ninguna modificación sustancial, sino de mero estilo lingüístico.

\section{III. ¿Qué es una sede de dirección?}

Si bien hemos arribado a una primera importante conclusión que es que la expresión "centro administrativo" (utilizada por la ONU) representa lo mismo que la expresión "sede de dirección" (utilizada por la OCDE), aún no hemos definido qué es exactamente una sede de dirección.

La pregunta resulta bastante curiosa debido a que los CMOCDE sólo se limitan a señalar que la sede de dirección es un ejemplo de lugar de negocios, al igual que una oficina, una sucursal, etc. Sin embargo, los CMOCDE no dan más luces acerca de los elementos definitorios de una sede de dirección que la distinguirían de otros lugares de negocios.

En nuestra opinión, la sede de dirección se asemeja a lo que la doctrina denomina "sede social" y que no puede confundirse con el concepto de "domicilio" de la persona jurídica. El tratadista Enrique Elías Laroza explica que "la sede social es entonces el lugar preciso en el que la sociedad puede realizar algunas de sus actividades o fijar su administración, mientras que el domicilio es la circunscripción territorial en cuyo Registro Público se inscribe la sociedad"3.

Similar diferencia es planteada por la autora Milka Aucca al señalar que "el domicilio social no es sinónimo de sede social. Con esta diferencia se quiere solucionar el problema que se genera cuando una sociedad tiene constituido su domicilio en una jurisdicción (por razones impositivas o económicas) y su sede principal en otra (por razones operativas)" 4 .

La citada autora, recurriendo a su vez a la doctrina especializada como Zaldívar,

2 GILDEMEISTER RUIZ-HUIDOBRO, Alfredo. “El establecimiento permanente como posible instrumento de integración en América Latina". Instituto Peruano de Derecho Tributario: Lima, 1996, página 51. Web: http://www.ipdt.org/editor/docs/02_Rev30_AGRH.pdf

3 ELÍAS LAROZA, Enrique. Derecho Societario Peruano. La Ley General de Sociedades del Perú. Tomo I. Gaceta Jurídica: Lima, 2015, página 130.

4 AUCCA ALVAREZ, Milka Irina. El mundo cambia, la empresa cambia: la movilidad societaria. Tesis para optar el título de Magister en Derecho de la Empresa. Pontificia Universidad Católica del Perú: Lima, 2015, página 26. 
Fargosi, Cornejo Costas, define a la sede social de la siguiente forma:

- Es aquella dirección específica dentro de una circunscripción. En ese sentido, la sociedad podrá contar con más de una sede o dirección social.

- Se entiende por sede social a aquel lugar preciso de una determinada ciudad o población donde funciona la administración y dirección de una sociedad.

- La sede social es donde la sociedad "funcionará", es la indicación detallada del lugar en donde regirá los órganos de gobierno, donde guardarán los libros, donde se llevará la gestión social.

Como puede observarse, la sede social es un concepto distinto del domicilio y está vinculado al lugar específico en que una persona jurídica desarrolla alguna de sus actividades o fija su administración, motivo por el cual una sola persona jurídica puede tener de forma simultaneo muchas sedes sociales.

En nuestra opinión, la sede de dirección sería un concepto equiparable al de sede social y, como tal, constituye un claro ejemplo de un lugar de negocios a la luz de la definición general de un establecimiento permanente.

\section{Sede de dirección efectiva}

Como hemos desarrollado anteriormente, para efectos de la determinación de un establecimiento permanente en el país, la sede de dirección representa lo mismo que un centro administrativo, con la única diferencia que la primera expresión es más utilizada por la comunidad tributaria internacional. Sin embargo, ¿podría considerarse que en realidad la intención del legislador al reemplazar la expresión "centro administrativo" fue incorporar el criterio de sede de dirección efectiva?

Naturalmente, si lo que se busca es hallar la intención del legislador, la Exposición de Motivos del Decreto Legislativo 1424 es el primer documento a revisar, lamentablemente en la exposición de motivos no se hace ninguna referencia sobre este punto. Sin embargo, teniendo en cuenta que la Exposición de Motivos hace referencia muchas veces a los trabajos de la OCDE, resultará relevante revisar qué señala dicha organización respecto a la sede de dirección efectiva.

Los CMOCDE anteriores a la versión del 2017 señalan que:

"La sede de dirección efectiva es el lugar donde se toman de hecho las decisiones comerciales clave y las decisiones de gestión necesarias para llevar a cabo el conjunto de las actividades empresariales o profesionales de la entidad. Para determinar la sede de dirección efectiva deben considerarse todos los hechos y circunstancias pertinentes".

Para efectos de determinar la sede de dirección efectiva de una entidad, se debe evaluar diversos factores, tales como dónde se celebran habitualmente las reuniones de su consejo de administración u órgano similar, desde donde se realiza la alta gestión cotidiana, donde ésta situada su oficina central, qué legislación nacional rige su situación jurídica, donde están archivados sus 
documentos contables, entre otros.

Ahora, como habíamos señalado anteriormente, si bien los CMOCDE no dan una definición acerca de qué debe entenderse por una sede de dirección, los CMOCDE sí la distinguen claramente de una sede de dirección efectiva al señalar que "Una entidad puede simultanear más de una sede de gestión, pero tendrá una única sede de dirección efectiva".

Sin embargo, lo anterior no es lo único que las diferencia, sino también su funcionalidad. La sede de dirección efectiva ha sido tradicionalmente concebida como una tie breaker rule, esto es, como una regla de solución de un conflicto de doble residencia por parte de una persona jurídica.

Así, antes del 2017, el MOCDE utilizaba el criterio sede de dirección efectiva para determinar donde una persona jurídica se consideraría residente para efectos de la aplicación de un CDI cuando, en virtud de la legislación de los Estados involucrados, la persona jurídica se consideraba residente en ambos Estados. Los CMOCDE anteriores al 2017 sostenían que, de esta manera, se dejaban de lado criterios puramente formales (ej. el de inscripción en un registro), para dar preferencia a un criterio sustancial, en el cual se da preferencia al Estado desde donde la persona jurídica se dirige efectivamente, esto es, donde las principales decisiones de dirección de la persona jurídica eran tomadas.

Actualmente, en los MOCDE del 2017 dicha funcionalidad ha sido relativizada y ahora se la utiliza como un criterio que, conjuntamente con otros, podría tomarse en cuenta para resolver un conflicto de doble residencia. En efecto, para resolver un conflicto de doble residencia, la OCDE se ha inclinado por un análisis casuístico, de manera que sean los Estados - a través de sus autoridades competentes- quienes decidan sobre la cuestión de la residencia de las personas jurídicas. Ello se materializaría a través de un procedimiento de acuerdo amistoso entre las autoridades competentes de los Estados involucrados, teniendo en cuenta, para su análisis, su sede de dirección efectiva, su lugar de constitución o de creación por otros procedimientos, así como cualquier otro factor pertinente. En ausencia de tal acuerdo, esa persona no podría acogerse a las reducciones o exenciones previstas en el CDI, salvo en la forma y medida que puedan acordar las autoridades competentes de los Estados involucrados.

Por su parte, la funcionalidad de la sede de dirección en la cláusula sobre establecimiento permanente está orientada a facilitar su identificación como un lugar de negocios, ya que se parte de la premisa que la sede de dirección por su propia naturaleza constituye un espacio físico en el país que se encuentra a disposición del sujeto no domiciliado. A diferencia de la sede de dirección efectiva, la sede de dirección no fue concebida como una tie breaker rule.

Como puede observarse, la sede de dirección y la sede de dirección efectiva tienen naturaleza distinta, siendo la primera un ejemplo de lo que es un lugar de negocios 
a luz de la definición general de un establecimiento permanente, mientras que la segunda actualmente es un criterio que se debe tomar en cuenta, junto con otros, para determinar la residencia de una persona jurídica que es considerada residente en dos Estados contratantes.

Por tanto, al tratarse de figuras jurídicas claramente diferenciables, podemos afirmar que en el Perú no se ha incorporado ni se ha pretendido incorporar el criterio de sede de dirección efectiva como un supuesto generador de establecimiento permanente.

Por último, téngase en cuenta que, si la intención del legislador hubiera sido incorporar un criterio de sede de dirección efectiva, entonces así lo hubiera expresado en la Exposición de Motivos del Decreto Legislativo 1424, toda vez que ello hubiera sido un cambio totalmente radical que hubiera ameritado una amplia explicación en la Exposición de Motivos. Sin embargo, nada de ello ocurrió, debido a que no se ha introducido un criterio de sede de dirección efectiva para determinar la existencia de un establecimiento permanente, sino que únicamente se reemplazó la expresión "centro administrativo" por el de "sede de dirección" al ser esta última la expresión más utilizada por la comunidad internacional y promovida por la OCDE, incluso utilizada por el Perú en sus CDI suscritos.

\section{Sede de dirección como establecimiento permanente}

Como hemos podido advertir, la sede de dirección en el país de un sujeto no domiciliado es un ejemplo de lo que constituye un lugar de negocios; sin embargo, la sede de dirección -por sí misma- no genera un establecimiento permanente del sujeto no domiciliado, sino que requiere que se cumplan con los otros dos requisitos que establece la definición general de establecimiento permanente, esto es, que dicho lugar de negocios sea fijo y que mediante el referido lugar fijo de negocios, el sujeto no domiciliado desarrolle, total o parcialmente, sus actividades.

Mal se haría en sostener que por el sólo hecho de que un sujeto no domiciliado tenga en el país una sede de dirección, entonces dicho supuesto -per sé- generaría un establecimiento permanente, toda vez que se estaría desconociendo que el listado lugares del numeral 1 del artículo 14-B de la LIR es meramente ejemplificativo de lugares que por su naturaleza calificarían como lugares de negocios, pero que requieren ser analizados a la luz de la definición general de establecimiento permanente para determinar si se constituyen como tal.

Similar posición adopta los CMOCDE al indicar que "dicho párrafo contiene una lista, no exhaustiva, de ejemplos que son lugares de negocios, los cuales pueden ser considerados como generadores de establecimiento permanente bajo el primer párrafo si es que cumplen los requisitos de dicho párrafo. Los ejemplos deben ser leídos en el contexto de la definición general dada en el primer párrafo. Los términos listados, "sede de dirección", "sucursal", "oficina", etc., deben ser interpretados en la manera de que dichos lugares constituirían establecimiento permanente solamente si cumplen los requisitos del primer párrafo [definición 
general de establecimiento permanente] y no son lugares de negocios en los que aplica el cuarto párrafo [actividades de naturaleza preparatoria o auxiliar]".

Teniendo en cuenta lo anterior, podemos concluir que la sede de dirección, al haberla conceptualizado como una sede social, es un claro ejemplo de un lugar de negocios, ya que es un lugar específico en el cual un sujeto no domiciliado desarrolla alguna de sus actividades o fija su administración. Dicha sede de dirección puede generar un establecimiento permanente en el Perú si es que tiene la condición de ser fijo y, además, mediante dicho lugar fijo de negocios en el país el sujeto no domiciliado desarrolla, total o parcialmente, sus actividades.

\section{Conclusión}

Como hemos desarrollado, la LIR no ha recogido el criterio de sede de dirección efectiva, sino el de sede de dirección como un ejemplo de un lugar de negocios para efectos de determinar la existencia de un establecimiento permanente en el país de un sujeto no domiciliado. Ello no representa un cambio sustancial respecto al término que el RLIR utiliza de "centro administrativo", ya que los mismos CMONU utilizan el término "centro administrativo" como símil de "sede de dirección" del MOCDE, siendo una cuestión de mero estilo lingüístico.

Por tanto, y por el momento, en el Perú no se ha agregado un nuevo criterio para determinar el domicilio de las personas jurídicas, manteniéndose el criterio formal del lugar de constitución. Sin embargo, bajo el panorama mundial actual y el camino trazado por el Perú para ingresar a la OCDE, no se puede descartar que a corto plazo se pueda introducir un criterio de sede de dirección efectiva, al igual que otros países de la región como México y Colombia (ambos países de la OCDE). 